\title{
Time Decay for Nonlinear Dissipative Schrödinger Equations in Optical Fields
}

\author{
Nakao Hayashi, ${ }^{1,2,3}$ Chunhua Li, ${ }^{1,2,3}$ and Pavel I. Naumkin ${ }^{1,2,3}$ \\ ${ }^{1}$ Department of Mathematics, Graduate School of Science, Osaka University, Osaka, Toyonaka 560-0043, Japan \\ ${ }^{2}$ Department of Mathematics, College of Science, Yanbian University, No. 977 Gongyuan Road, Yanji, Jilin 133002, China \\ ${ }^{3}$ Centro de Ciencias Matemáticas, UNAM Campus Morelia, AP 61-3 (Xangari), 58089 Morelia, MICH, Mexico
}

Correspondence should be addressed to Chunhua Li; sxlch@ybu.edu.cn

Received 11 December 2015; Accepted 18 January 2016

Academic Editor: Yao-Zhong Zhang

Copyright (c) 2016 Nakao Hayashi et al. This is an open access article distributed under the Creative Commons Attribution License, which permits unrestricted use, distribution, and reproduction in any medium, provided the original work is properly cited.

We consider the initial value problem for the nonlinear dissipative Schrödinger equations with a gauge invariant nonlinearity $\lambda|u|^{p-1} u$ of order $p(n)<p \leq 1+2 / n$ for arbitrarily large initial data, where the lower bound $p(n)$ is a positive root of $(n+2) p^{2}-6 p-n=$ 0 for $n \geq 2$ and $p(1)=1+\sqrt{2}$ for $n=1$. Our purpose is to extend the previous results for higher space dimensions concerning $\mathbf{L}^{2}$-time decay and to improve the lower bound of $p$ under the same dissipative condition on $\lambda \in \mathbb{C}: \operatorname{Im} \lambda<0$ and $|\operatorname{Im} \lambda|>((p-1) / 2 \sqrt{p})|\operatorname{Re} \lambda|$ as in the previous works.

\section{Introduction and Main Results}

We consider the initial value problem for the following nonlinear Schrödinger equations:

$$
\begin{aligned}
i \partial_{t} u+\frac{1}{2} \Delta u & =\lambda|u|^{p-1} u, \quad(t, x) \in \mathbb{R} \times \mathbb{R}^{n}, \\
u(0, x) & =u_{0}(x), \quad x \in \mathbb{R}^{n},
\end{aligned}
$$

where $\lambda \in \mathbb{C}, \operatorname{Im} \lambda<0,|\operatorname{Im} \lambda|>((p-1) / 2 \sqrt{p})|\operatorname{Re} \lambda|$ and $1<p \leq 1+2 / n$. From the physical point of view, (1) is applied to investigate the light traveling in optical fibers (see, e.g., $[1,2])$. Some new nonlinear works concerning the related equations appear in $[3,4]$. We note that $\operatorname{Im} \lambda<$ 0 implies the dissipation of $|u(t, x)|$ by nonlinear Ohm's law (see, e.g., [5]). Therefore, the dissipative nonlinearity causes the transportation of the energy. The condition on the coefficient $\lambda$ appeared in the previous papers $[1,2,6,7]$ and so forth. It yields a dissipative property of solutions in one space dimension for arbitrarily large initial data, which was shown in [2]. In this paper, we prove $\mathbf{L}^{2}$-time decay estimate of solutions in any space dimension $n \geq 1$ under the condition of critical or subcritical power order nonlinearity such that

$$
p(n)<p \leq 1+\frac{2}{n},
$$

where

$$
\begin{aligned}
& p(n)=\frac{3+\sqrt{9+n^{2}+2 n}}{n+2} \text { for } n \geq 2, \\
& p(1)=1+\sqrt{2} \quad \text { for } n=1 .
\end{aligned}
$$

In [2], $\mathbf{L}^{\infty}$-time decay of solutions was studied in one space dimension under the condition on $p$ such that $2.686 \approx(5+$ $\sqrt{33}) / 4<p \leq 3$. More precisely, in [2] it was shown that the estimates of solutions to (1),

$$
\begin{aligned}
& \|u(t)\|_{L^{\infty}} \leq C t^{-1 / 2}(\log t)^{-1 / 2} \quad \text { for } p=3, \\
& \|u(t)\|_{L^{\infty}} \leq C t^{-1 /(p-1)} \text { for } \frac{5+\sqrt{33}}{4}<p<3,
\end{aligned}
$$

hold for any $t>1$ in the case of $n=1$. For small solutions, in [1] under the conditions such that $\operatorname{Im} \lambda<0$ and $p$ is close to $1+2 / n$, the same $\mathbf{L}^{\infty}$-time decay as stated above 
was obtained for $1 \leq n \leq 3$. Below we show that if we restrict our attention to the $\mathrm{L}^{2}$-time decay of solutions to (1) for arbitrarily large initial data, then we can reduce the low bound of the power $p$ and consider the problem in higher space dimensions. To investigate the effect of the dissipative nonlinearity, we consider the estimates about the $\mathbf{L}^{2}$ norm of solutions to (1). In [2] the $\mathbf{L}^{2}$-time decay of the solution $u$ to (1) satisfying that $\lim _{t \rightarrow \infty}\|u(t)\|_{\mathbf{L}^{2}}=0$ was obtained, when $n=1$ and $(21+\sqrt{177}) / 12<p \leq 3$. We develop our problem to the space dimension $n \geq 1$. Moreover the scope of $p$ is extended, when we focus on one space dimension case. Another point in this paper is to prove results directly comparing to the contradiction argument in [2]. More precisely, our strategy of the proof is as follows. We first transform our target equation (1) into the following nonlinear ordinary equation with remainder terms $R$ :

$$
\partial_{t} f-g(t) f^{p} \leq C|R|
$$

where

$$
\begin{aligned}
g(t) & =(\operatorname{Im} \lambda) t^{-(n / 2)(p-1)}, \\
f & =|\mathscr{F} U(-t) u|
\end{aligned}
$$

(see (48) and (69)). Next by using the positive solution of the separate equation $\partial_{t} F-g(t) F^{p}=0$ with $F(0)=\left|\widehat{u_{0}}\right|$, we translate it into the separate equation such that

$$
\partial_{t}\left(F^{-p} f\right) \leq\left(\frac{p-1}{p}\right) p g(t)+C F^{-p}|R|,
$$

by using the condition $\operatorname{Im} \lambda<0$ and the Young inequality (see (56)). The desired estimate of $f$ is obtained by integration in time through the estimate of $F$.

By $\mathbf{L}^{p}$ we denote the usual Lebesgue space with the norm

$$
\|\phi\|_{L^{p}}=\left(\int_{\mathbb{R}^{n}}|\phi(x)|^{p} d x\right)^{1 / p}
$$

if $1 \leq p<\infty$ and

$$
\|\phi\|_{\mathbf{L}^{\infty}}=\underset{x \in \mathbb{R}^{n}}{\operatorname{ess} . \sup }|\phi(x)|
$$

For $m, s \in R$, weighted Sobolev space $\mathbf{H}^{m, s}$ is defined by

$$
\mathbf{H}^{m, s}=\left\{\phi \in \mathbf{L}^{2} ;\|\phi\|_{\mathbf{H}^{m, s}}<\infty\right\},
$$

where

$$
\|\phi\|_{\mathbf{H}^{m, s}}=\left\|(1-\Delta)^{m / 2}\left(1+|x|^{2}\right)^{s / 2} \phi\right\|_{\mathbf{L}^{2}} .
$$

We write $\|\phi\|_{\mathbf{L}^{2}}=\|\phi\|$ and $\mathbf{H}^{m, 0}=\mathbf{H}^{m}$ for simplicity.

Let us introduce some notations. We define the dilation operator by

$$
\left(D_{t} \phi\right)(x)=\frac{1}{(i t)^{n / 2}} \phi\left(\frac{x}{t}\right)
$$

and define $M=e^{(i / 2 t)|x|^{2}}$ for $t \neq 0$. Free evolution group $\mathcal{U}(t)$ is written as

$$
\mathcal{U}(t)=M D_{t} \mathscr{F} M
$$

where the Fourier transform of $\phi$ is

$$
(\mathscr{F} \phi)(\xi)=\frac{1}{(2 \pi)^{n / 2}} \int_{\mathbb{R}^{n}} e^{-i x \cdot \xi} \phi(x) d x .
$$

We also have

$$
\mathcal{U}(-t)=\bar{M} \mathscr{F}^{-1} D_{t}^{-1} \bar{M},
$$

where the inverse Fourier transform

$$
\left(\mathscr{F}^{-1} \phi\right)(x)=\frac{1}{(2 \pi)^{n / 2}} \int_{\mathbb{R}^{n}} e^{i x \cdot \xi} \phi(\xi) d \xi .
$$

These formulas were used in [8] first to study the asymptotic behavior of solutions to nonlinear Schrödinger equations. We denote by the same letter $C$ various positive constants. by

The standard generator of Galilei transformation is given

$$
\mathscr{J}(t)=\mathscr{U}(t) x \mathcal{U}(-t)=x+i t \nabla .
$$

We have commutation relations with $\mathscr{J}$ and $\mathscr{L}=i \partial_{t}+(1 / 2) \Delta$ such that

$$
[\mathscr{L}, \mathscr{J}]=0 .
$$

To prove Theorem 1, we introduce the function space

$$
\begin{aligned}
X_{1, T} & =\{u ; \mathcal{U}(-t) u \\
& \left.\in C\left([0, T) ; \mathbf{H}^{0,1} \cap \mathbf{H}^{1}\right),\|u\|_{X_{1, T}}<\infty\right\},
\end{aligned}
$$

where

$$
\|u\|_{X_{1, T}}=\sup _{0 \leq t<T}\|\mathcal{U}(-t) u(t)\|_{\mathbf{H}^{0,1} \cap \mathbf{H}^{1}} .
$$

In Theorems 1 and 2, we consider the subcritical and critical cases, respectively. Define

$$
\begin{aligned}
& p(n)=\frac{3+\sqrt{9+n^{2}+2 n}}{n+2} \text { for } n \geq 2, \\
& p(1)=1+\sqrt{2} \text { for } n=1
\end{aligned}
$$

Theorem 1. Assume that $\lambda \in \mathbb{C}, \operatorname{Im} \lambda<0, \quad|\operatorname{Im} \lambda|>((p-$ $1) / 2 \sqrt{p})|\operatorname{Re} \lambda|$, and $1<p<\infty$, if $n=1,2$, and $1<p<$ $(n+2) /(n-2)$, if $n \geq 3, u_{0} \in \mathbf{H}^{0,1} \cap \mathbf{H}^{1}$. Then (1) has a unique global solution $u \in X_{1, \infty}$. Moreover if we assume that

$$
p(n)<p<1+\frac{2}{n} \quad \text { for } n \geq 1
$$

then we have

$$
\|u(t)\|_{\mathbf{L}^{2}} \leq C\langle t\rangle^{-(1 /(p-1)-n / 2) q}
$$

for $t \geq 0$, where $0 \leq q \leq 2 n /(n+2)$ and the identity

$$
|\operatorname{Im} \lambda| \int_{0}^{\infty} \int_{\mathbb{R}^{n}}|u|^{p+1} d x d \tau=\frac{1}{2}\left\|u_{0}\right\|_{\mathrm{L}^{2}}^{2}
$$

holds. 
The lower bound $p(n)$ in Theorem 1 is approximated as

$$
\begin{aligned}
& p(1)=1+\sqrt{2} \approx 2.414, \\
& p(2)=\frac{1}{4}(3+\sqrt{17}) \approx 1.7808, \\
& p(3)=\frac{1}{5}(3+\sqrt{24}) \approx 1.5798 .
\end{aligned}
$$

Since $p(1)<(21+\sqrt{177}) / 12$ holds, where $(21+\sqrt{177}) / 12$ was introduced in [2] to study the $\mathbf{L}^{2}$-estimates of the solution $u(t, x)$ for $(1)$, the lower bound of $p$ is improved.

We next consider the critical case $p=1+2 / n$.

Theorem 2. Let $u$ be the solution of (1) with $p=1+2 / n$ stated in Theorem 1. Then we have

$$
\|u(t)\|_{\mathbf{L}^{2}} \leq C(\log t)^{-(n / 2) q}
$$

fort $>2$, where $0 \leq q \leq 2 n /(n+2)$ and the identity (24) holds.

\section{Proof of Theorem 1}

Under the assumptions

$$
\begin{aligned}
\operatorname{Im} \lambda & <0, \\
|\operatorname{Im} \lambda| & >\frac{p-1}{2 \sqrt{p}}|\operatorname{Re} \lambda|,
\end{aligned}
$$

we have a dissipative property of solutions. Indeed we have by the usual energy method

$$
\begin{aligned}
& \frac{1}{2} \partial_{t}\|\nabla u\|^{2}=\operatorname{Im}\left(\lambda \int_{\mathbb{R}^{n}} \nabla\left(|u|^{p-1} u\right) \cdot \overline{\nabla u} d x\right), \\
& \frac{1}{2} \partial_{t}\|\mathscr{J} u\|^{2}=\operatorname{Im}\left(\lambda \int_{\mathbb{R}^{n}} \mathscr{J}\left(|u|^{p-1} u\right) \cdot \overline{\mathscr{J} u} d x\right) .
\end{aligned}
$$

Nonlinear terms are represented as

$$
\begin{aligned}
& \operatorname{Im}\left(\lambda \int_{\mathbb{R}^{n}} \nabla\left(|u|^{p-1} u\right) \cdot \overline{\nabla u} d x\right) \\
& =\operatorname{Im}\left(\lambda \frac{p+1}{2} \int_{\mathbb{R}^{n}}|u|^{p-1}|\nabla u|^{2} d x\right) \\
& \quad+\operatorname{Im}\left(\lambda \frac{p-1}{2} \int_{\mathbb{R}^{n}}|u|^{p-3} u^{2}(\overline{\nabla u})^{2} d x\right) \\
& \leq\left(\frac{p+1}{2} \operatorname{Im} \lambda+\frac{p-1}{2}|\lambda|\right) \int_{\mathbb{R}^{n}}|u|^{p-1}|\nabla u|^{2} d x \\
& \leq 0
\end{aligned}
$$

if $\operatorname{Im} \lambda<0$ and

$$
\frac{p-1}{2}|\lambda| \leq \frac{p+1}{2}|\operatorname{Im} \lambda|
$$

which is equivalent to

$$
|\operatorname{Im} \lambda| \geq \frac{p-1}{2 \sqrt{p}}|\operatorname{Re} \lambda| .
$$

Therefore we have

$$
\begin{aligned}
& \frac{1}{2}\|\nabla u\|^{2}-C(\lambda) \int_{0}^{t} \int_{\mathbb{R}^{n}}|u|^{p-1}|\nabla u|^{2} d x d \tau \\
& \quad \leq \frac{1}{2}\left\|\nabla u_{0}\right\|^{2}
\end{aligned}
$$

for $t \geq 0$, where

$$
C(\lambda)=\frac{p+1}{2} \operatorname{Im} \lambda+\frac{p-1}{2}|\lambda| .
$$

In the same way as in the proof of (29), we have

$$
\begin{aligned}
& \operatorname{Im}\left(\lambda \int_{\mathbb{R}^{n}} \mathscr{J}\left(|u|^{p-1} u\right) \cdot \overline{\mathscr{J} u} d x\right) \\
& =\operatorname{Im}\left(\lambda \frac{p+1}{2} \int_{\mathbb{R}^{n}}|u|^{p-1}|\mathscr{J} u|^{2} d x\right) \\
& \quad-\operatorname{Im}\left(\lambda \frac{p-1}{2} \int_{\mathbb{R}^{n}}|u|^{p-3} u^{2}(\overline{\mathscr{J} u})^{2} d x\right) \\
& \leq C(\lambda) \int_{\mathbb{R}^{n}}|u|^{p-1}|\mathscr{J} u|^{2} d x \leq 0,
\end{aligned}
$$

from which it follows that

$$
\begin{aligned}
& \frac{1}{2}\|\mathscr{J} u\|^{2}-C(\lambda) \int_{0}^{t} \int_{\mathbb{R}^{n}}|u|^{p-1}|\mathcal{J} u|^{2} d x d \tau \\
& \quad \leq \frac{1}{2}\left\|x u_{0}\right\|^{2}
\end{aligned}
$$

for $t \geq 0$. We also have

$$
\frac{1}{2}\|u(t)\|_{\mathrm{L}^{2}}^{2}+|\operatorname{Im} \lambda| \int_{0}^{t} \int_{\mathbb{R}^{n}}|u|^{p+1} d x d \tau=\frac{1}{2}\left\|u_{0}\right\|^{2}
$$

Therefore by (32), (35), and (36), we have an a priori estimate of solutions

$$
\|u\|^{2}+\|\nabla u\|^{2}+\|\mathscr{J} u\|^{2} \leq\left\|u_{0}\right\|^{2}+\left\|\nabla u_{0}\right\|^{2}+\left\|x u_{0}\right\|^{2},
$$

which implies the global in time existence of solutions to (1) in the function space $X_{1, \infty}$ for $\operatorname{Im} \lambda<0$ and $|\operatorname{Im} \lambda|>((p-$ $1) / 2 \sqrt{p})|\operatorname{Re} \lambda|$. This completes the first part of the proof of the theorem.

Our concern is to estimate the time decay rate in the subcritical case since it is expected that the decay rate of solutions is different from that of solutions to linear problem. Denote $\varphi=\mathscr{F} \mathscr{U}(-t) u$, and $v=\mathscr{F} M \mathscr{U}(-t) u$. Using the 
factorization formula $\mathcal{U}(t)=M D_{t} \mathscr{F} M$, we multiply both sides of (1) by $\mathscr{F} \mathscr{U}(-t)$ to get

$$
\begin{aligned}
& \mathscr{F} \mathcal{U}(-t)|u|^{p-1} u=\mathscr{F} \bar{M} \mathscr{F}^{-1} D_{t}^{-1} \bar{M}|u|^{p-1} u \\
&= t^{-(n / 2)(p-1)} \mathscr{F} \bar{M} \mathscr{F}^{-1}\left|D_{t}^{-1} \bar{M} u\right|^{p-1} D_{t}^{-1} \bar{M} u \\
&= t^{-(n / 2)(p-1)} \mathscr{F} \bar{M} \mathscr{F}^{-1}|v|^{p-1} v \\
&= t^{-(n / 2)(p-1)}|\varphi|^{p-1} \varphi \\
&+t^{-(n / 2)(p-1)}\left(|v|^{p-1} v-|\varphi|^{p-1} \varphi\right) \\
&+t^{-(n / 2)(p-1)} \mathscr{F}(\bar{M}-1) \mathscr{F}^{-1}|v|^{p-1} v \\
&= t^{-(n / 2)(p-1)}|\varphi|^{p-1} \varphi+R,
\end{aligned}
$$

where

$$
\begin{aligned}
R= & t^{-(n / 2)(p-1)}\left(|v|^{p-1} v-|\varphi|^{p-1} \varphi\right) \\
& +t^{-(n / 2)(p-1)} \mathscr{F}(\bar{M}-1) \mathscr{F}^{-1}|v|^{p-1} v .
\end{aligned}
$$

For the remainder term $R$, we have by (37) the following.

Lemma 3. Let $u$ be a solution of (1) in the function space $X_{1, \infty}$. Then the estimate

$$
\|R(t)\|_{\mathbf{L}^{2}} \leq C t^{-(n / 2)(p-1)-(1 / 2) s}\left\|u_{0}\right\|_{\mathbf{H}^{0,1}}^{p}
$$

for $t \geq 1$ is true, where

$$
\begin{aligned}
s= & \frac{n}{2}-\frac{n-2}{2} p \\
& \text { for } n \geq 3,0<s<1 \text { for } n=2, s=1 \text { for } n=1 .
\end{aligned}
$$

Proof. By Hölder's and Sobolev's inequalities, we get

$$
\begin{aligned}
\| \mathscr{F} & (\bar{M}-1) \mathscr{F}^{-1}|v|^{p-1} v \|_{\mathbf{L}^{2}} \\
& \leq C t^{-(1 / 2) s}\left\||\nabla|^{s}|v|^{p-1} v\right\|_{\mathbf{L}^{2}} \\
& \leq C t^{-(1 / 2) s}\left\||v|^{p-1}\right\|_{\mathbf{L}^{n /(1-s)}}\left\||x|^{s} \mathcal{U}(-t) u\right\|_{\mathbf{L}^{q}} \\
& \leq C t^{-(1 / 2) s}\|v\|_{\mathbf{L}^{(n /(1-s))(p-1)}}^{p-1}\||x| \mathcal{U}(-t) u\|_{\mathbf{L}^{2}} \\
& \leq C t^{-(1 / 2) s}\left(\|u\|^{p-1}+\|\mathscr{J} u\|^{p-1}\right)\|\mathscr{J} u\|,
\end{aligned}
$$

where $s=1$ for $n=1,0<s<1$ for $n=2$, and

$$
s=\frac{n}{2}-\frac{n-2}{2} p \quad \text { for } n \geq 3,
$$

since we can apply the Sobolev embedding theorem with

$$
\begin{aligned}
\frac{1}{q} & =\frac{1}{2}-\frac{1-s}{n}, \\
\frac{1-s}{n(p-1)} & \geq \frac{1}{2}-\frac{1}{n} .
\end{aligned}
$$

In the same manner with the same $s$ as above, we obtain

$$
\begin{aligned}
& \left\||v|^{p-1} v-|\varphi|^{p-1} \varphi\right\|_{L^{2}} \\
& \leq C\left\|\left(|v|^{p-1}+|\varphi|^{p-1}\right)(v-\varphi)\right\|_{L^{2}} \\
& \quad=C\left\|\left(|v|^{p-1}+|\varphi|^{p-1}\right) \mathscr{F}(M-1) \mathcal{U}(-t) u\right\|_{\mathbf{L}^{2}} \\
& \leq C\left(\left\||v|^{p-1}\right\|_{\mathbf{L}^{n /(1-s)}}+\left\||\varphi|^{p-1}\right\|_{\mathbf{L}^{n /(1-s)}}\right) \\
& \cdot\|\mathscr{F}(M-1) \mathcal{U}(-t) u\|_{\mathbf{L}^{q}} \\
& \leq C\left(\|v\|_{\mathbf{L}^{(n /(1-s))(p-1)}}^{p-1}+\|\varphi\|_{\mathbf{L}^{(n /(1-s))(p-1)}}^{p-1}\right) \\
& \cdot\left\||\nabla|^{1-s} \mathscr{F}(M-1) \mathcal{U}(-t) u\right\|_{\mathbf{L}^{2}} \\
& \leq C t^{-(1 / 2) s}\|v\|_{\mathbf{L}^{(n /(1-s))(p-1)}}^{p-1}\||x| \mathcal{U}(-t) u\|_{\mathbf{L}^{2}} \\
& \leq C t^{-(1 / 2) s}\left(\|u\|^{p-1}+\|\mathscr{J} u\|^{p-1}\right)\|\mathscr{J} u\| .
\end{aligned}
$$

In view of (37) the result of the lemma follows. This completes the proof of the lemma.

We continue to prove Theorem 1 . We let $\varphi=\mathscr{F} \mathcal{U}(-t) u$. Then we have the ordinary differential equation

$$
i \partial_{t} \varphi=\lambda t^{-(n / 2)(p-1)}|\varphi|^{p-1} \varphi+\lambda R .
$$

Multiplying both sides of (46) by $\bar{\varphi}$ and taking the imaginary part of the resulting equation, we obtain

$$
\frac{1}{2} \partial_{t}|\varphi|^{2}=(\operatorname{Im} \lambda) t^{-(n / 2)(p-1)}|\varphi|^{p+1}+\operatorname{Im}(\lambda R \bar{\varphi})
$$

from which it follows that

$$
\partial_{t}|\varphi|-(\operatorname{Im} \lambda) t^{-(n / 2)(p-1)}|\varphi|^{p} \leq C|R|
$$

Let us consider the case $R=0$. Namely, we consider the separate equation

$$
(\operatorname{Im} \lambda)^{-1} t^{(n / 2)(p-1)} F^{-p} d F-d t=0 .
$$

It is well known that a solution of the separate equation (49) containing an arbitrary constant is obtained by integration. Then we have the solution

$$
F(t, \xi)=\frac{\left|\widehat{u_{0}}(\xi)\right| K}{\left(\left|\widehat{u_{0}}(\xi)\right|^{p-1} t^{1-(n / 2)(p-1)}+K^{(p-1)}\right)^{1 /(p-1)}},
$$

where

$$
K=\left(\frac{1-(n / 2)(p-1)}{|\operatorname{Im} \lambda|(p-1)}\right)^{1 /(p-1)}
$$

Therefore we have the estimate for $0 \leq q \leq 1$

$$
|F(t, \xi)| \leq C\left|\widehat{u_{0}}(\xi)\right|^{1-q} t^{(n / 2-1 /(p-1)) q} .
$$


Hence for the solution of (49) we have the estimate

$$
\begin{aligned}
\|F(t)\|_{\mathbf{L}^{2}} & \leq C\left\|{\widehat{u_{0}}}_{0}\right\|_{\mathbf{L}^{2(1-q)}}^{1-q} t^{(n / 2-1 /(p-1)) q} \\
& \leq C\left\|u_{0}\right\|_{\mathbf{H}^{1,0}}^{1-q} t^{(n / 2-1 /(p-1)) q}
\end{aligned}
$$

if $2 /(n+2) \geq q$. It suggests to us that the solution of (47) has the same asymptotic profile, when $R$ is considered as the remainder term. We now change (48) into a separate form by using the solution of (47). Multiplying both sides of (48) by $F^{-p}$ we obtain

$$
\begin{aligned}
\partial_{t}\left(F^{-p}|\varphi|\right) \\
\leq|\operatorname{Im} \lambda| t^{-(n / 2)(p-1)}\left(p F^{-1}|\varphi|-\left(F^{-1}|\varphi|\right)^{p}\right) \\
\quad+C F^{-p}|R| .
\end{aligned}
$$

By the Young inequality $|a||b| \leq(1 / p)|a|^{p}+(1 / q)|b|^{q}$ with $1 / p+1 / q=1$, we get

$$
\begin{aligned}
p F^{-1}|\varphi| & =\left(p^{1 / p} F^{-1}|\varphi|\right) p^{1-1 / p} \\
& \leq\left(F^{-1}|\varphi|\right)^{p}+\left(\frac{p-1}{p}\right) p .
\end{aligned}
$$

We apply this estimate to the first term of the right-hand side of (54) to have

$$
\begin{aligned}
d\left(F^{-p}|\varphi|\right) \leq & \left(\frac{p-1}{p}\right) p|\operatorname{Im} \lambda| t^{-(n / 2)(p-1)} d t \\
& +C F^{-p}|R| d t .
\end{aligned}
$$

This is the separable form of (48). Integrating in time and using (50), we arrive at

$$
\begin{aligned}
F^{-p}(t, \xi)|\varphi(t, \xi)| \leq & F^{-p}(1, \xi)|\varphi(1, \xi)|+C t^{\alpha} \\
& +C \int_{1}^{t} s^{(\alpha /(p-1)) p}|R(s, \xi)| d s
\end{aligned}
$$

where $\alpha=1-(n / 2)(p-1)$, which implies

$$
\begin{aligned}
& |\varphi(t, \xi)| \\
& \quad \leq C F^{p}(t, \xi)\left(t^{\alpha}+\int_{1}^{t} s^{(\alpha /(p-1)) p}|R(s, \xi)| d s\right) .
\end{aligned}
$$

Hence by

$$
F^{p}(t, \xi) \leq C t^{-\alpha} \frac{\left|\widehat{u_{0}}(\xi)\right|}{\left(\left|\widehat{u_{0}}(\xi)\right|^{p-1} t^{1-(n / 2)(p-1)}+1\right)^{1 /(p-1)}},
$$

we have for $0 \leq q \leq 1$

$$
\begin{aligned}
& |\varphi(t, \xi)| \leq C\left|\widehat{u_{0}}(\xi)\right|^{1-q} t^{-(1 /(p-1))(1-(n / 2)(p-1)) q} \\
& \quad+C t^{-(1 /(p-1))(1-(n / 2)(p-1)) q} t^{-\alpha} \int_{1}^{t} \tau^{(\alpha /(p-1)) p}|R(\tau, \xi)| d \tau .
\end{aligned}
$$

Taking $\mathbf{L}^{2}$ norm and applying Lemma 3, we get for $q \leq 2 /(n+$ 2)

$$
\begin{aligned}
\|\varphi(t)\|_{\mathbf{L}^{2}} & \\
\leq & C\left\|u_{0}\right\|_{\mathbf{H}^{1}}^{1-q} t^{-(\alpha /(p-1)) q} \\
& +C t^{-(\alpha /(p-1)) q} t^{-\alpha} \int_{1}^{t} \tau^{(\alpha /(p-1)) p}\|R(\tau)\|_{\mathbf{L}^{2}} d \tau \\
\leq & C t^{-(\alpha /(p-1)) q} \\
& +C t^{-(\alpha /(p-1)) q} t^{-\alpha} \int_{2}^{t} \tau^{(\alpha /(p-1)) p-(n / 2)(p-1)-(1 / 2) s} d \tau \\
\leq & C t^{-(\alpha /(p-1)) q}\left(1+t^{\alpha /(p-1)-(n / 2)(p-1)-(1 / 2) s+1}\right) .
\end{aligned}
$$

We have

$$
t^{\alpha /(p-1)-(n / 2)(p-1)-(1 / 2) s+1} \longrightarrow 0 \text { as } t \longrightarrow \infty
$$

if $p$ satisfies

$$
\begin{aligned}
& p>p(n)=\frac{3+\sqrt{9+n^{2}+2 n}}{n+2} \text { for } n \geq 2, \\
& p>p(1)=1+\sqrt{2} .
\end{aligned}
$$

Therefore we find that

$$
\|\varphi(t)\|_{L^{2}} \leq C t^{-(\alpha /(p-1)) q}=C t^{(n / 2-1 /(p-1)) q} .
$$

This is the nonlinear version of estimate (53). We now prove the time decay of solutions. We have the formula

$$
\begin{aligned}
u(t) & =\mathscr{U}(t) \mathcal{U}(-t) u=M D_{t} \mathscr{F} M \mathscr{U}(-t) u \\
& =M D_{t} \varphi+M D_{t} \mathscr{F}(M-1) \mathcal{U}(-t) u,
\end{aligned}
$$

where $\varphi=\mathscr{F} U(-t) u$. By a direct computation

$$
\left\|M D_{t} \varphi\right\|_{\mathbf{L}^{2}}=\|\varphi\|_{\mathbf{L}^{2}}
$$

Hence by (64) and the estimate

$$
\begin{aligned}
& \left\|M D_{t} \mathscr{F}(M-1) \mathcal{U}(-t) u\right\|_{\mathrm{L}^{2}} \leq C t^{-1 / 2}\|\mathscr{J} u\|_{\mathrm{L}^{2}} \\
& \quad \leq C t^{-1 / 2}\left\|x u_{0}\right\|_{\mathrm{L}^{2}},
\end{aligned}
$$

which follows from (35), we have

$$
\begin{aligned}
\|u(t)\|_{\mathbf{L}^{2}} & \leq\|\varphi(t)\|_{\mathbf{L}^{2}}+C t^{-1 / 2}\|\mathscr{J} u\|_{\mathbf{L}^{2}} \\
& \leq C t^{(n / 2-1 /(p-1)) q}+C t^{-1 / 2}\left\|x u_{0}\right\|_{\mathbf{L}^{2}} \\
& \leq C t^{(n / 2-1 /(p-1)) q}
\end{aligned}
$$

since $p>1+2 /(1+n)$, and we find $t^{-1 / 2}<t^{n / 2-1 /(p-1)}$ for $t \geq 1$. Therefore, identity (24) follows from (68) and (36). 


\section{Proof of Theorem 2}

We start with

$$
\partial_{t}|\varphi|-(\operatorname{Im} \lambda) t^{-1}|\varphi|^{1+2 / n} \leq C|R| .
$$

Note that the solution of the separable equation

$$
\partial_{t} F-(\operatorname{Im} \lambda) t^{-1} F^{1+2 / n}=0
$$

is represented as

$$
\begin{aligned}
F(t, \xi)=\frac{\left|\widehat{u_{0}}(\xi)\right| K}{\left(\left|\widehat{u_{0}}(\xi)\right|^{2 / n}(\log t)+K^{2 / n}\right)^{n / 2}} & \\
K & =\frac{n^{n / 2}}{(2|\operatorname{Im} \lambda|)^{n / 2}} .
\end{aligned}
$$

Multiplying both sides of (69) by $F^{-1-2 / n}$, we obtain

$$
\begin{aligned}
\partial_{t}\left(F^{-1-2 / n}|\varphi|\right) & \\
\leq & |\operatorname{Im} \lambda| t^{-1}\left(\frac{n+2}{n} F^{-1}|\varphi|-F^{-1-2 / n}|\varphi|^{1+2 / n}\right) \\
& +C F^{-1-2 / n}|R| .
\end{aligned}
$$

Applying the Young inequality to the first term of the lefthand side of the above inequality, we obtain

$$
\begin{aligned}
& \frac{n+2}{n} F^{-1}|\varphi| \\
& \quad=\left(\left(\frac{n+2}{n}\right)^{n /(n+2)} F^{-1}|\varphi|\right)\left(\frac{n+2}{n}\right)^{2 /(n+2)} \\
& \quad \leq F^{-1-2 / n}|\varphi|^{1+2 / n}+\frac{2}{n} .
\end{aligned}
$$

Hence we obtain the separate form of (69) such that

$$
d\left(F^{-1-2 / n}|\varphi|\right) \leq \frac{2}{n}|\operatorname{Im} \lambda| t^{-1} d t+C F^{-1-2 / n}|R| d t .
$$

Integrating in time, we obtain

$$
\begin{aligned}
F^{-1-2 / n}(t)|\varphi(t)| \leq & F^{-1-2 / n}(2)|\varphi(2)|+C \log t \\
& +C \int_{2}^{t}(\log \tau)^{(n+2) / 2}|R| d \tau
\end{aligned}
$$

from which it follows that

$$
|\varphi(t)| \leq C F^{1+2 / n}(t)\left(\log t+\int_{2}^{t}(\log \tau)^{(n+2) / 2}|R| d \tau\right) .
$$

Since

$$
F^{1+2 / n}(t) \leq \frac{\left|\widehat{u_{0}}(\xi)\right| K}{\left(\left|\widehat{u_{0}}(\xi)\right|^{2 / n}(\log t)+K^{2 / n}\right)^{n / 2}}(\log t)^{-1}
$$

we have

$$
\begin{aligned}
|\varphi(t)| \leq & C\left|\widehat{u_{0}}(\xi)\right|^{1-q}(\log t)^{-(n / 2) q} \\
& +C(\log t)^{-1-n / 2} \int_{2}^{t}(\log \tau)^{(n+2) / 2}|R| d \tau
\end{aligned}
$$

for any $0 \leq q \leq 1$. We obtain by Lemma 3

$$
\begin{aligned}
& \|\varphi(t)\|_{\mathbf{L}^{2}} \\
& \leq C\left\|u_{0}\right\|_{\mathbf{H}^{1}}^{1-q}(\log t)^{-(n / 2) q} \\
& \quad+C(\log t)^{-1-n / 2} \int_{2}^{t}(\log \tau)^{(n+2) / 2} \tau^{-1-(1 / 2) s} d \tau \\
& \leq C\left\|u_{0}\right\|_{\mathbf{H}^{1}}^{1-q}(\log t)^{-(n / 2) q}+C(\log t)^{-1-n / 2} \\
& \leq C(\log t)^{-(n / 2) q}
\end{aligned}
$$

for $t>2$. The rest of the proof is the same as that of Theorem 1 . This completes the proof of Theorem 2 .

\section{Conflict of Interests}

The authors declare that there is no conflict of interests regarding the publication of this paper.

\section{Acknowledgments}

The work of Nakao Hayashi is partially supported by JSPS KAKENHI Grant Nos. 25220702 and 15H03630. The work of Chunhua $\mathrm{Li}$ is partially supported by the Education Department of Jilin Province ([2015] No. 34) and NNSFC under Grant No. 11461074. The work of Pavel I. Naumkin is partially supported by CONACYT and PAPIIT Project IN100113.

\section{References}

[1] N. Kita and A. Shimomura, "Asymptotic behavior of solutions to Schrödinger equations with a subcritical dissipative nonlinearity," Journal of Differential Equations, vol. 242, no. 1, pp. 192-210, 2007.

[2] N. Kita and A. Shimomura, "Large time behavior of solutions to Schrödinger equations with a dissipative nonlinearity for arbitrarily large initial data," Journal of the Mathematical Society of Japan, vol. 61, no. 1, pp. 39-64, 2009.

[3] W.-R. Sun, B. Tian, Y. Jiang, and H.-L. Zhen, "Optical rogue waves associated with the negative coherent coupling in an isotropic medium," Physical Review E, vol. 91, no. 2, Article ID 023205, 2015

[4] X.-Y. Xie, B. Tian, W.-R. Sun, and Y. Sun, "Rogue-wave solutions for the Kundu-Eckhaus equation with variable coefficients in an optical fiber," Nonlinear Dynamics, vol. 81, no. 3, pp. 1349-1354, 2015.

[5] G. P. Agrawal, Nonlinear Fiber Optics, Academic Press, 1995.

[6] V. A. Liskevich and M. A. Perelmuter, "Analyticity of submarkovian semigroups," Proceedings of the American Mathematical Society, vol. 123, no. 4, pp. 1097-1104, 1995. 
[7] N. Okazawa and T. Yokota, "Global existence and smoothing effect for the complex Ginzburg-Landau equation with $p$ laplacian," Journal of Differential Equations, vol. 182, no. 2, pp. 541-576, 2002.

[8] N. Hayashi and T. Ozawa, "Scattering theory in the weighted $\mathrm{L}^{2}\left(\mathbb{R}^{n}\right)$ spaces for some Schrödinger equations," Annales de l'Institut Henri Poincaré, Physique Théorique, vol. 48, pp. 17-37, 1988. 


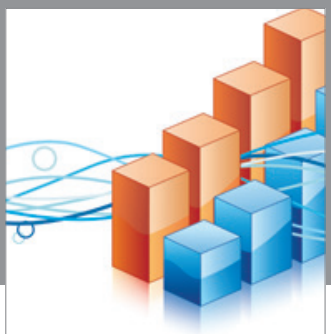

Advances in

Operations Research

vatem alat4

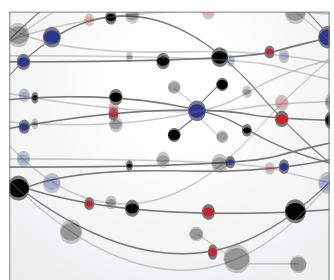

\section{The Scientific} World Journal
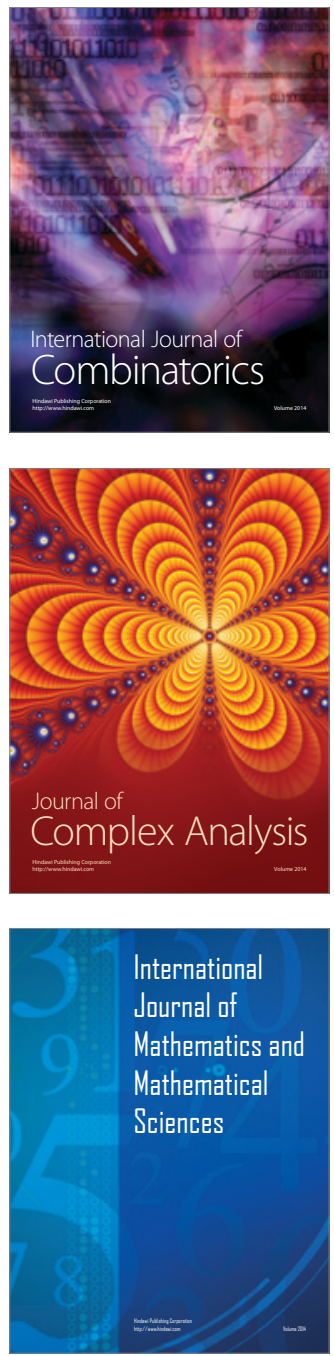
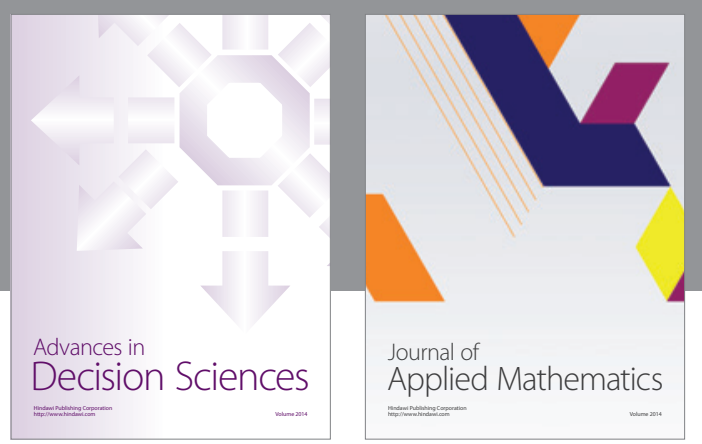

Algebra

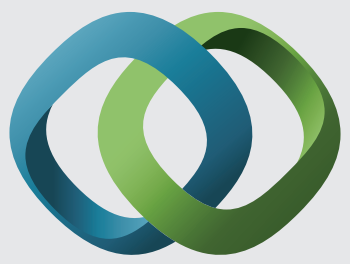

\section{Hindawi}

Submit your manuscripts at

http://www.hindawi.com
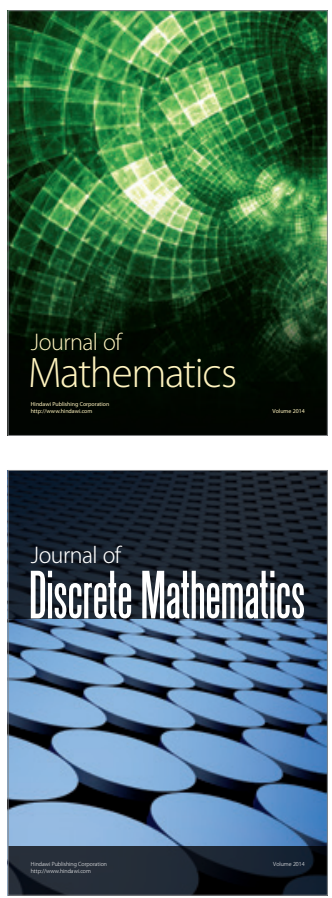

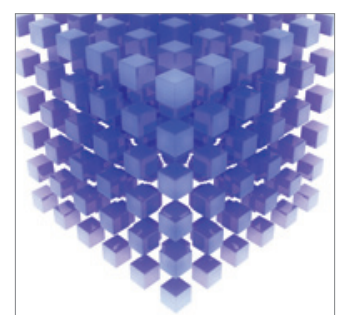

Mathematical Problems in Engineering
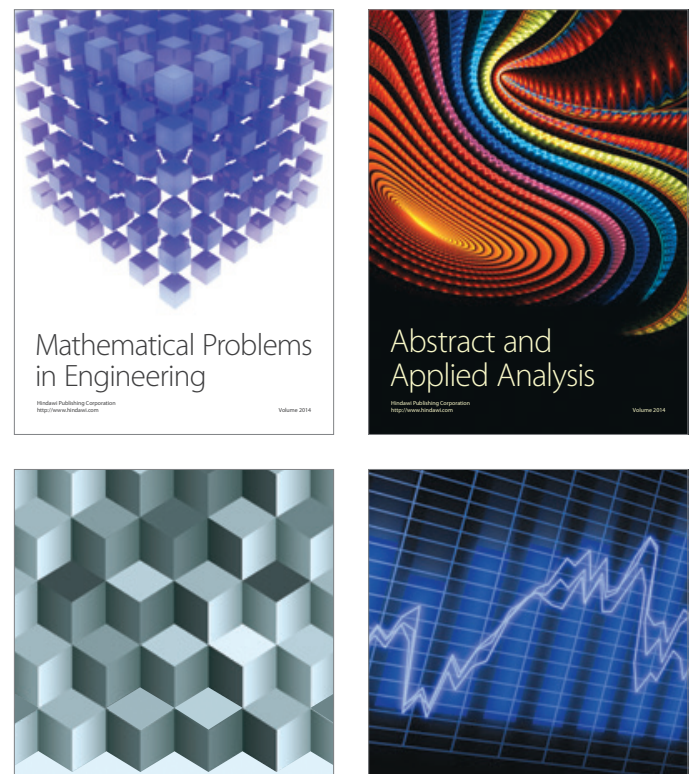

Journal of

Function Spaces

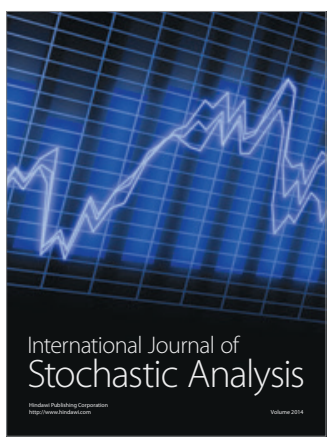

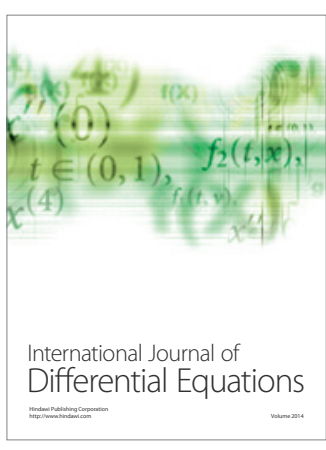
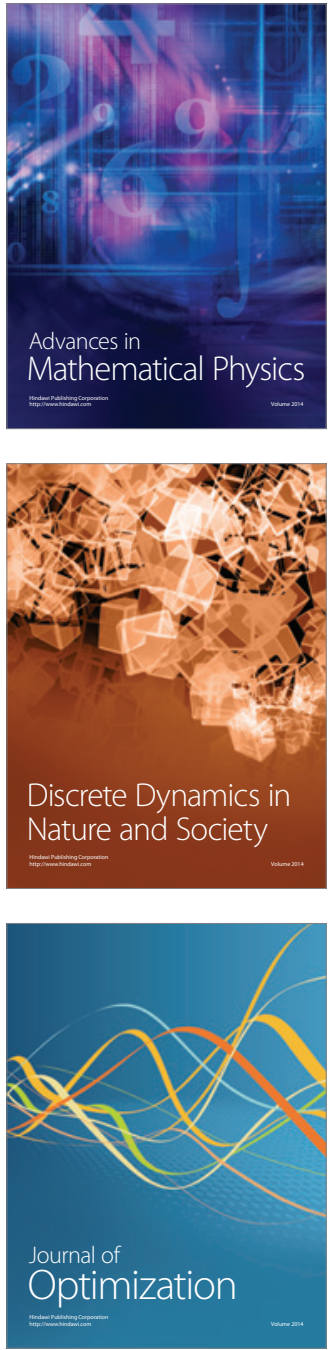